\title{
Twelve hundred non-triggered gamma ray bursts
}

\author{
B. Stern ${ }^{1,4}$, Ya. Tikhomirova ${ }^{2}$, M. Stepanov ${ }^{3}$, D. Kompaneets ${ }^{2}$, A. Berezhnoy ${ }^{3}$, and R. Svensson ${ }^{4}$ \\ 1 Institute for Nuclear Research, Russian Academy of Sciences, Moscow 117312, Russia \\ e-mail: stern@al20.inr.troitsk.ru \\ 2 Astro Space Center of Lebedev Physical Institute, Moscow, Profsoyuznaya 84/32, 117810, Russia \\ 3 Skobeltsyn Institute of Nuclear Physics, Moscow State University, Moscow 119899, Russia \\ ${ }^{4}$ Stockholm Observatory, S-133 36 Saltsjöbaden, Sweden
}

Received January 21; accepted June 25, 1999

\begin{abstract}
We present preliminary results of an off-line search for non-triggered gamma-ray bursts (GRBs) in the BATSE daily records for about 5.7 years of observations. We found more GRB-like events than the yield of the similar search of Kommers et al. (1998) and extended the $\log N-\log P$ distribution down to $\sim 0.1 \mathrm{ph} \mathrm{cm}^{-2} \mathrm{~s}^{-1}$. The indication of a turnover of the $\log N-\log P$ at a small $P$ is not confirmed: the distribution is straight at 1.5 decades with the power law index -.6 and cannot be fitted with a standard candle cosmological model.
\end{abstract}

Key words: gamma-ray bursts — methods: data analysis

\section{Introduction}

Many gamma-ray bursts which were too weak to cause the BATSE to trigger or were being missed due to other reasons (data readouts etc.) can be confidently identified in the BATSE daily records which cover the full period of the CGRO operation.

The systematic search for non-triggered GRBs was started by Kommers et al. (1997). Recently, Kommers et al. (1998) completed the data scan for 6 years.

Despite that we started our search (in November, 1997) for non-triggered bursts much later than Kommers et al. (1997), the motivation of the work was that we started our scan with some important advances. Firstly, we used a more selective off-line trigger code. Secondly, and most importantly, is the method of measurement of the efficiency of the GRB search using artificial test bursts that we employed.

\section{Data scan}

We use $1024 \mathrm{~ms}$ time resolution BATSE data (DISCLA) from the ftp archive of the Goddard Space Flight Center at ftp://cossc.gsfc.nasa.gov/compton/data/batse/daily/.
The procedure of data reduction contains the following steps:

Step 1. Conversion of the original BATSE records adding to them artificial test bursts prepared from real rescaled bursts taken from the BATSE database.

Test bursts were added to the data at a random time with an average time interval 25000 s (i.e., the number of test bursts exceeds the number of real bursts).

\section{Step 2. Data scan}

We performed automatic check for the trigger conditions. Each trigger was followed by a human decision whether the trigger is a GRB candidate. The person performing the scan was unaware whether it was a real or a test burst. All candidates were recorded as a fragments of daily records saving all original information.

\section{Step 3. Event classification.}

The candidate events were discarded or classified as non-GRBs if they do not satisfy a number of criteria.

Step 4. Separation of tests bursts using the protocols generated on step 1 .

\section{Preliminary results}

We scanned 2068 days of BATSE daily records and found 1243 non-triggered events which can be classified as classic GRBs (Kommers et al. 1998 found 837 non-triggered GRBs per 2200 days). We found 1374 bursts which were triggered by BATSE (Kommers et al. 1998 detected 1393 BATSE triggered events), and missed near 350 BATSE triggers: some of them are in data gaps, some are too short to be detected with $1 \mathrm{~s}$ time resolution. We also found 3780 test bursts out of about 6800 added to the data. All test bursts were identified and excluded from the final sample. The peak flux distribution of real events found in the scan and classified as GRBs is presented in Fig. 1. 


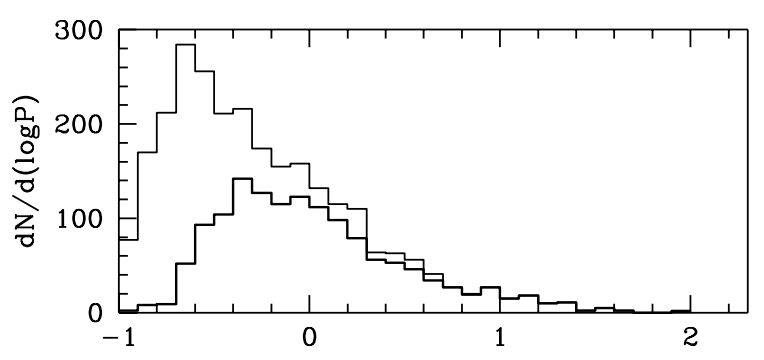

Fig. 1. Differential peak flux distribution of detected GRBs. Thick histogram - the distribution of BATSE triggers detected in the scan (1374). Thin histogram - all bursts detected in the scan (2617). Both distributions are given before correction for the efficiency is applied

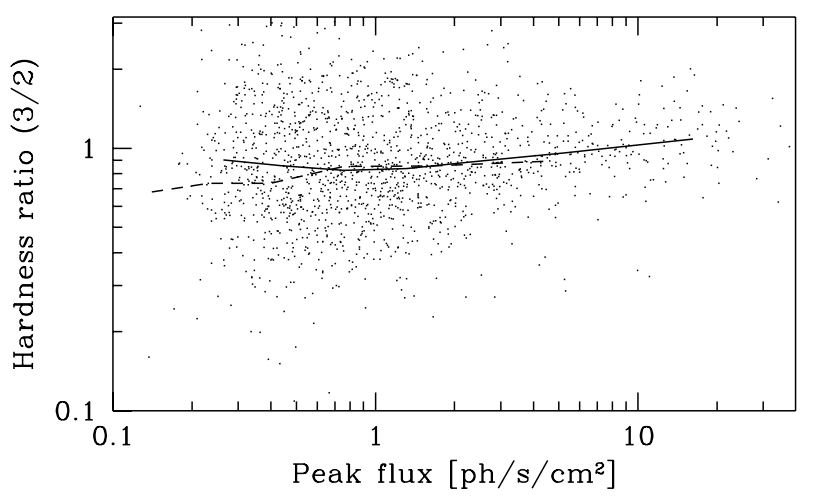

Fig. 2. Hardness - peak flux plot for all detected GRBs. Solid line - median value of the hardness ratio for triggered GRBs; dotted line - the same for non-triggered GRBs

Data gaps and periods of a high ionospheric background are taken into account so the efficiency is normalized to whole elapsed time of CGRO operation.

The hardness - peak flux scattering plot shown in Fig. 2 demonstrates that new weak bursts give a direct continuation of the distribution of stronger GRBs. The well-known brightness-hardness correlation (Mallozi et al. 1995; Nemiroff et al. 1994), is clearly visible. Nemiroff et al. (1994) found the hardness ratio for $3 / 2$ channels to vary by factor 1.54 from weak to strong bursts $(64 \mathrm{~ms}$ resolution peak flux). In our case of 1024 ms resolution many short hard bursts fall to the weak end of the distribution reducing the correlation. With the same reason weak triggered bursts are slightly harder than nontriggered bursts. Nevertheless we see a reasonable global correlation where median hardness varies by factor 1.58 in approximate agreement with Nemiroff et al. (1994).

The resulting $\log N-\log P$ distribution in absolute units is presented in Fig. 3 in comparison with BATSE $\log N-\log P$ from Meegan et al. (1998) (in arbitrary normalisation) and that from Kommers et al. (1998) (in absolute units).

We fit the $\log N-\log P$ distributions with the simplest hypothesis of the standard candle non-evolving GRB population: the best fit gives $\chi^{2}=50.2$ at 22 degrees of free-

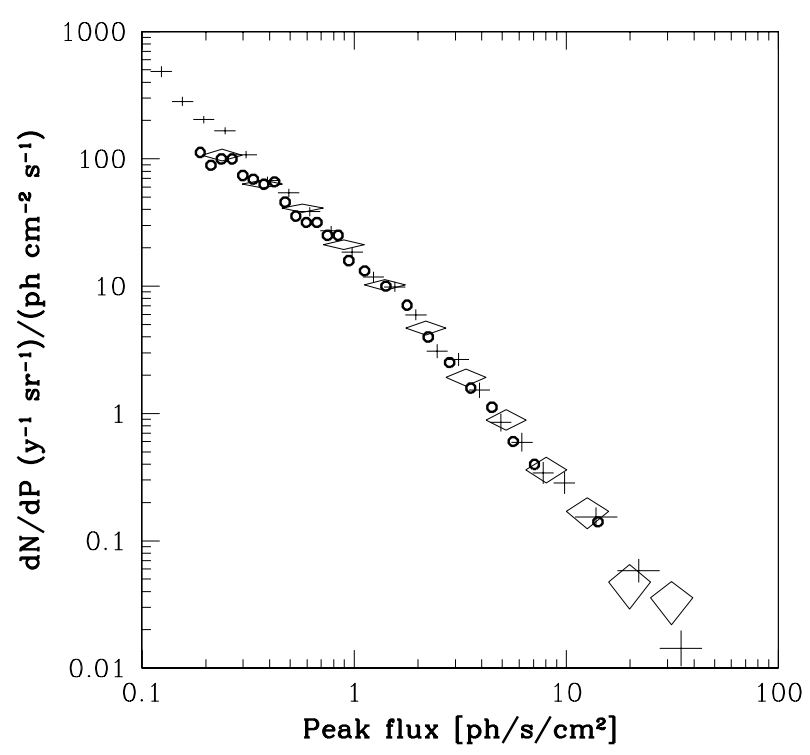

Fig. 3. The differential $\log N-\log P$ distribution in absolute units for all events (2617) (1243 non-triggered) detected in the scan. Data of Kommers et al. (1998) are shown by circles, BATSE-3 distribution from Meegan et al. (1998) is shown by diamonds (in arbitrary normalisation). Our data are corrected for the "test bursts" efficiency which is $\sim 0.72$ for bright bursts (due to data gaps and bad background intervals) and smoothly declines to the lowest peak fluxes

dom. For the first time the simplest cosmological model cannot fit data. The fit will be even worse if we use the star formation rate curve as the GRB evolution scenario. The rejection of the standard candle hypothesis is not surprising, however this is still an achievement because the cosmological fit of the $\log N-\log P$ becomes conclusive.

Acknowledgements. We thank Aino Skassyrskaia, Andrei Skorbun, Eugeni Stern, Vladimir Kurt, Kirill Semenkov, Stas Masolkin, Alex Sergeev, Max Voronkov and Felix Ryde for valuable assistance. We acknowledge Andrei Beloborodov and Juri Poutanen for useful discussions.

This work was supported by the Swedish Natural Science Research Council, the Royal Swedish Academy of Science, the Wennergren Foundation for Scientific Research, and a NORDITA Nordic Collaboration Project grant. RFBR grant 97-02-16975 supported one of the authors (D.K.).

\section{References}

Bagot, Zwart, Yungel'son, 1998, A\&A 332, L57

Kommers J., et al., 1997, ApJ 491, 704

Kommers J., et al., 1998, astro-ph/9809300

Mallozzi R.S., Pendleton G.N., Paciesas W.S., 1995, ApJ 454, 597

Meegan C., et al., 1998, in Gamma Ray Bursts, AIP Conf. Proc. 428, 3

Nemiroff R.J., et al., 1994, ApJ 435, L133

Stern B., Poutanen J., Svensson R., 1997, ApJ 489, L41

Totani T., 1997, ApJ 486, L71 